\title{
Vibrotactile loudness addition
}

JAMES C. CRAIG

PRINCETON UNIVERSITY

Os adjusted the intensity of vibration at a single locus on the right hand to a value equal in vibratory loudness to various patterns of vibration on the left hand. The patterns were created by 1 to 5 equated vibration generators, varied with respect to sensation level and distances among the vibrators. The results were: (a) increasing from 1 to 5 vibrators produced a doubling in vibratory loudness, (b) neither loudness level of the components nor distance among vibrators had any effect on the slope of the overall loudness growth function. Os also adjusted the intensity of a white noise to equal in magnitude the patterns of vibration presented (a) to the left hand as before and (b) to loci distributed over the surface of the body. The results were the same as those obtained using a single vibrator as standard. The specific loci stimulated did not appear to have any effect on vibrotactile loudness addition.

The growth of sensory magnitude or "loudness" of vibration has typically been studied using a single stimulus source. For example, the effect of intensity or amplitude of vibration on cutaneous loudness judgments has been investigated by Spector (1953) and Stevens (1959b), the effects of duration on vibratory loudness by Békésy (1939), of frequency by Gibson (1959), and of locus by Békésy (1960, p. 566) and Stevens (1959b).

Békésy has described several observations on multiple stimulation of the skin and its effect on sensory magnitude. For one such observation, Békésy (1960, pp. 596-599) placed five vibrators at frequencies ranging from 20 to $320 \mathrm{~Hz}$ on the surface of the lower arm, $2 \mathrm{~cm}$ apart. All five vibrators were adjusted for equal loudness. Following the onset of stimulation, all five vibrators could be felt at their respective frequencies and loci. However, after $10 \mathrm{sec} .$, the whole pattern flowed together to produce a sensation at the center of the pattern with a "pitch" equal to that of the center vibrator. While only the pitch of the center vibrator could be appreciated, all five vibrators contributed to the loudness of the pattern, since turning off the end vibrators produced no change in pitch but did produce a reduction in loudness. As Békésy put it, "Thus up to a certain distance from the locus of the sensation we have 'pitch' inhibition with a simultaneous summation of magnitude" (Békésy, 1960, p. 597).

The direct impetus for the present study was an observation growing out of an experiment by Geldard and Sherrick (1965) in which 10 vibrators were placed at different loci over the surface of the body. One vibrator was set at $15 \mathrm{~dB}$ sensation level (SL), and the other nine vibrators were matched to this vibrator for equal loudness. The loudness produced by a single vibrator was not great; but when all ten vibrators were energized simul- taneously, the overall "feel" of the resulting pattern was very much more noticeable than was that of any one vibrator. As the 10 vibrators were turned on, Os reported a "squeezing" sensation of great magnitude (Geldard, 1966).

The method for attacking this problem of vibrotactile loudness addition was suggested by several similar studies done in the field of audition. Fletcher and Munson (1933) and Howes (1950) studied loudness addition of multicomponent tones. Howes had Os adjust the loudness of a standard $1000-\mathrm{Hz}$ tone to equal the loudness of complexes of tones involving from 1 to 11 equally loud tones. In general, Howes found, as had Fletcher and Munson, that the observed loudness of a complex tone increased linearly with the number of tones making up the complex. Doubling the number of tones in a complex produced a doubling in the loudness, provided that the components were not $70 \mathrm{~dB}$ SPL or more.

The present study was undertaken to investigate the manner in which multiple vibrotactile stimuli summate in loudness and some of the factors that affect this summation.

\section{EXPERIMENT I}

From the work in audition, one would predict that if vibrotactile loudness addition were ideal, i.e., followed the addition of energy, doubling the number of equally loud vibrators in a pattern should produce a doubling in the loudness of the pattern.

\section{Apparatus and Procedure}

A diagram of the apparatus, originally described by Geldard and Sherrick (1965), is shown in Fig. 1. The purpose of the apparatus was to control the number of vibrators energized, the loci on the skin that were to be stimulated, the duration of stimulation, the interstimulus interval, and the intensity of stimulation. Tektronix equipment controlled repetition rate, interstimulus interval, and stimulus duration. The number and position of the vibrators energized were determined by two Western Union Model 1.A tape transmitters.

One of the tape transmitters controlled the switching of five vibrators, the test vibrators, attached to the finger tips of the left hand of an $\mathrm{O}$. The other tape transmitter switched a Grason-Stadler model E 3262 A recording attenuator that controlled the input to a Goodmans model $V-47$ vibrator, the standard vibrator. A foot switch connected to the attenuator permitted Os to manipulate the signal level of the standard. The standard vibrator had a round contactor tip $2 \mathrm{~cm}$ in diameter, and the test vibrators were of an inertial type designed and described by Sherrick (1965). These weré coupled 


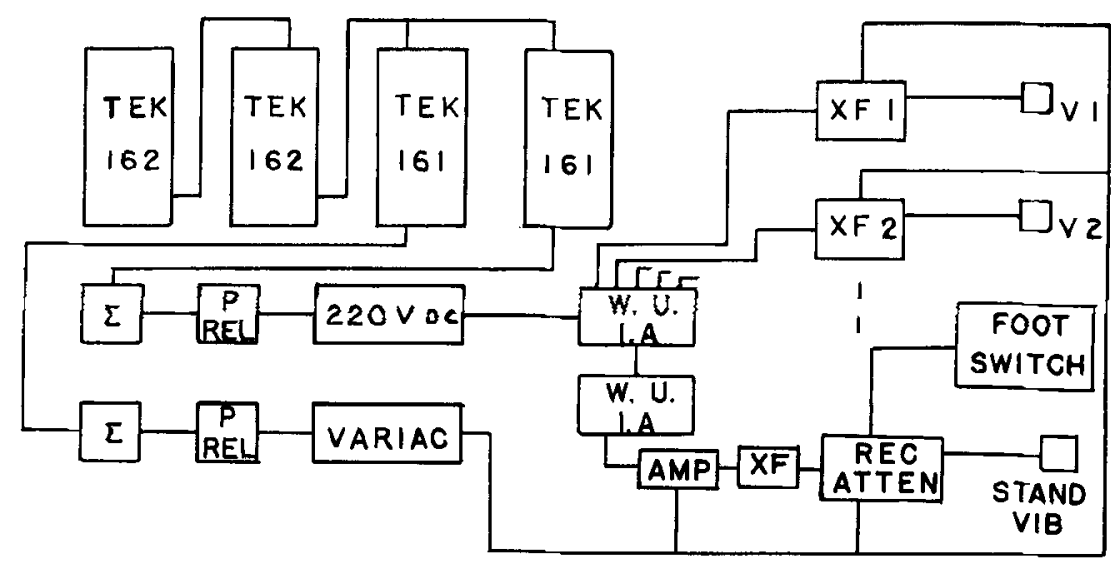

Fig. 1. Block diagram of the apparatus.

to the O's left finger tips by means of plastic rings that attached to brass rods screwed to the base of the vibrators. In order to prevent transmission of vibration between them, the vibrators were cradled by rubber bands attached to metal plates. The plates in turn were fastened to the ceiling of the room by lucite rods and rubber tubing couplers.

Three experienced Os served throughout the experiments. During the experimental sessions, the $O$ was seated in an IAC model 404-A sound isolation booth and fitted with earphones through which a masking white noise was fed from a Grason-Stadler model 455-B noise generator. With his right middle finger tip on the contactor of the standard vibrator, $O$ received a $200-\mathrm{msec}$. burst of $60-\mathrm{Hz}$ vibration at the rate of 1 per sec. $O$ was instructed to adjust the amplitude of vibration continuously from the level at which he could feel it all the time to the level at which he could never feel it. The mean of three of these adjustments was the absolute threshold above which the amplitude of the standard vibrator was increased $15 \mathrm{~dB} . \mathrm{E}$ then turned on the first test vibrator to present a burst of $60-\mathrm{Hz}$ vibration of $200-\mathrm{msec}$. duration simultaneously with the standard vibrator set at 15 dB SL. E increased the amplitude of the test vibrator until $\mathrm{O}$ judged it to be equal in vibratory loudness to the standard vibrator. This procedure was repeated for test vibrators two through five, after which the entire equation procedure was repeated to allow for shifts in $\mathrm{O}^{\prime} \mathrm{s}$ initial criteria.

In the main portion of the session O adjusted the loudness of the standard vibrator by means of the attenuator foot switch control to equal the loudness of the test pattern on the left hand. The stimuli were presented in the following order: a 200-msec. burst of a test pattern, an 800-msec. blank period, a 200-msec. burst from the standard vibrator, a 2800-msec. blank period. The sequence was repeated as many times as necessary for $O$ to be satisfied with his match. The $\mathrm{dB}$ SL set on the standard vibrator was computed from the setting on the recording attenuator. $O$ was instructed to move the attenuator to either a very high or very low setting between trials to prevent sequential effects among trials.

The fingers were numbered from left to right (little finger $=1$; thumb $=5$ ). The test patterns were: vibrator number $3 ; 5 ; 1,5 ; 4,5 ; 1,3,5 ; 2,3,4 ; 1,2,4,5 ; 1,2,3,4$; and two patterns with all five vibrators. Patterns with two, three, and four vibrators were chosen to provide two extremes of distance among patterns, close and far. An experimental session consisted of the presentation of these ten patterns without regard to order.

\section{Results and Discussion}

Nine matches were made for each pattern. An analysis of the data revealed no significant differences in magnitude between close and far patterns having the same number of stimuli. Because there was no difference between them, the results from both close and far patterns were combined to yield the mean values presented in Fig. 2. A slope of 9.1 was computed from the log-log plot of SL of the standard vs. number of vibrators.

In his 1950 study, Howes measured the loudness of the standard tone in sones, a scale of sensory magnitude used in audition. Howes' results, plotted as Iog sones vs. log number of tones in the component, yielded a function with a slope of unity.

In order to compare more easily the results of the present study with Howes' work, a scale of subjective intensity of vibration, similar to the sone scale, was needed. The scale was also needed because, as Stevens indicates, using a matching technique alone it is not possible "to learn the form of the function relating sensory response to stimulus intensity" (Stevens, 1959b, p. 216). Fortunately, in the present case, the growth of the sensory magnitude of vibration has already been studied using the method of magnitude estimation (Stevens, $1959 \mathrm{~b})$. The slope resulting from the plotting of log sensory magnitude estimations against $\mathrm{dB}$ of amplitude was 0.96 . Increasing the intensity of vibration $6 \mathrm{~dB}$ produced an approximate doubling of the loudness of vibration. The data from Fig. 2 were replotted in Fig. 3 with $10 g$ subjective intensity plotted against log number of components. The resulting slope, 0.43 , shows that doubling the number of vibrators in a pattern does not produce a doubling in loudness. In fact, an increase in number from one to five vibrators in a pattern is needed to produce a doubling in loudness. 


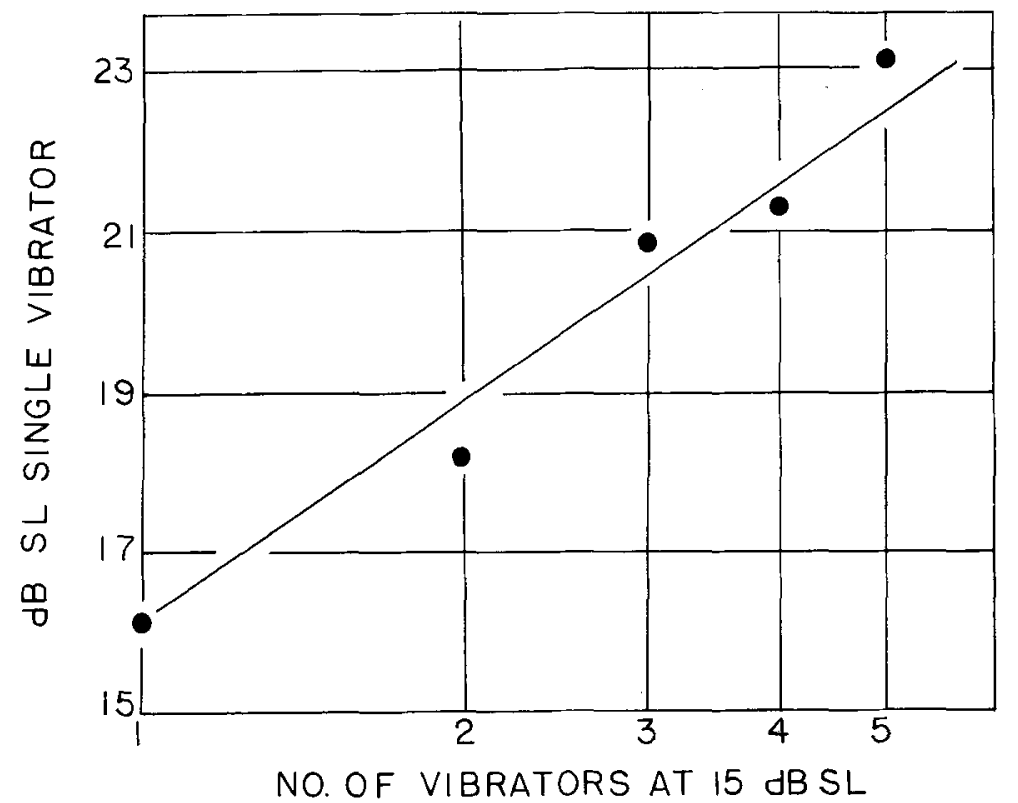

Fig. 2. Loudness match of single vibrator to different numbers of vibrators.

\section{EXPERIMENT II}

In the Howes (1950) and the Fletcher and Munson (1933) studies, two conditions could prevent ideal summation of loudness in complex tones: (a) increasing the components to $70 \mathrm{~dB}$ SPL or more and (b) decreasing the frequency differences between tones. The slope of the summation function for these loud tones decreased progressively from unity as the loudness level was increased, and pitch differences between tones were reduced from 500 mels to $250 \mathrm{mels}$. Howes speculated that this loss in loudness summation may be due to greater physical interaction along the basilar membrane among tones that are loud and close together.

By analogy, loudness of the individual vibrators and distance among vibrators should have an effect on loudness addition. Because distance among vibrators did not produce any effect on vibrotactile loudness addition in Experiment I, the loudness level of the individual vibrators was chosen as the more promising of the two dimensions as a contributor to the observed reduction in loudness addition. In this experiment two additional loudness levels were tested, 10 and $25 \mathrm{~dB}$ SL. If vibration at separate loci on the skin interacts in a manner similar to that of tones on the basilar membrane, one would predict that the slope produced by the vibrators set at $10 \mathrm{~dB}$ SL would be greater than the slope value of 9.1 found at $15 \mathrm{~dB}$ SL. Moreover, the slope at $25 \mathrm{~dB}$ SL would be less than 9.1, showing a decrease in loudness addition.

\section{Apparafus and Procedure}

The apparatus and procedure were the same as in Experiment I, except that the standard vibrator was set at $10 \mathrm{~dB}$ SL for the test vibrators to be matched to it for one set of observations, and at $25 \mathrm{~dB}$ SL for another set.

\section{Results and Discussion}

As in Experiment I, an analysis of the data revealed no significant differences between close and far patterns at either $10 \mathrm{~dB}$ SL or $25 \mathrm{~dB}$ SL. When the means of 18 observations per point are plotted, the slope of the function relating a single vibrator to patterns of vibrators at $10 \mathrm{~dB}$ SL is 8.0 ; at $25 \mathrm{~dB}$ SL the slope is 8.6. Neither of the two slopes differs significantly from the slope at $15 \mathrm{~dB}$ SL of 9.1 ; moreover, the ordering of the magnitude of the slopes is not in the manner predicted by analogy from the Howes' study. Thus, the only effect of changing the loudness level of each component of the pattern is to move the plot up or down the ordinate while leaving the slope of the function unchanged.

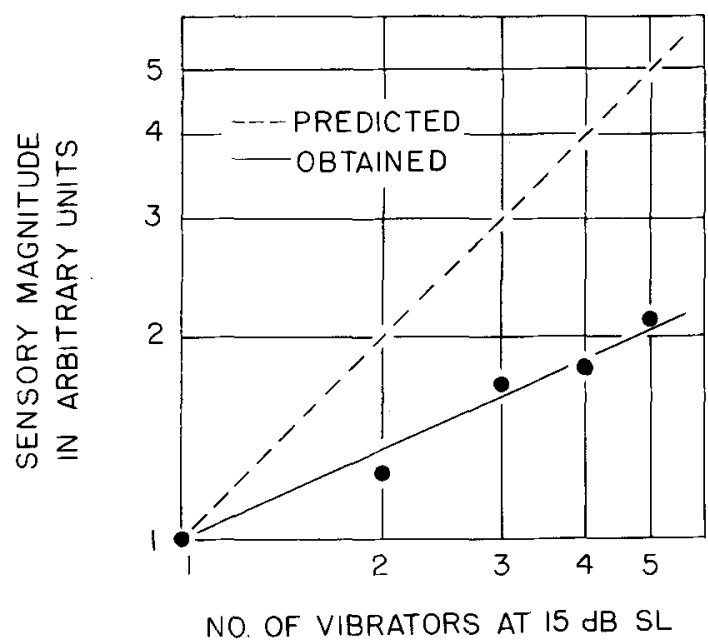

Fig. 3. Sensory magnitude of different numbers of vibrators. Increase from 1 to 2 arbitrary units indicates a doubling in sensory magnitude. Predicted line has a slope value equal to 1. 


\section{EXPERIMENT III}

In Experiments I and II the test vibrators were located in a relatively confined area, the left hand. Because of this, it was possible for Os to attend to the entire pattern as a whole and to match the magnitude of the pattern with the standard vibrator. However, preliminary observations indicated that when the test vibrators were shifted from the finger tips to more widely dispersed areas on the body, Os had considerable difficulty matching the test patterns with a single vibrator. Os reported that the presence of the single vibrator caused them to focus attention upon a single locus of stimulation and made it difficult to attend to the overall patterns distributed on the surface of the body. For this reason, pulsed white noise led to the ears was tried as a standard. White noise had the virtue that, like multiple vibrotactile stimulation, it seemed to spread out and surrouni the body. Also, enough was known about the growth of sensory magnitude of auditory white noise for a check to be made on the reliability of such a match.

J. C. Stevens and E. Tulving (1957) had had Os make magnitude estimations of the loudness of different intensities of white noise and had obtained a slope of 0.6 from a $\log -\log$ plot. Subsequently, S. S. Stevens (1959a), who obtained a slope of 0.96 from a log-log plot of magnitude estimations of vibration applied to the finger tip, predicted that if Os were asked to adjust the loudness of a white noise to equal the loudness of a vibration or vice versa, the result of a $\log -\log$ plot of $\mathrm{dB}$ of vibration against $\mathrm{dB}$ of white noise should equal 0.6 divided by 0.96 or approximately 0.6 . Carrying out the match, Stevens found that the slope did equal 0.6.

In Experiment I of this study, the slope resulting from the match of the loudness of a standard vibrator to the loudness of one or more vibrators set at $15 \mathrm{~dB}$ SL on the finger tips was 9.1. One would predict that if Os were asked to set a level of white noise equal to the cutaneous loudness of a pattern vibration on the finger tips with one or more vibrators in it, the slope of a log-log plot would be 9.1 divided by 0.6 or 15.2 .

\section{Apparatus and Procedure}

In the first part of this experiment the apparatus and procedure were the same as in Experiment I. After seting the standard vibrator at $25 \mathrm{~dB}$ SL and adjusting the test vibrators on the left hand to equal it in loudness, the standard was removed from the circuit. The output of a Grason-Stadler model 455-B noise generator was led to a relay which was energized by one of the Tektronix pulse generators. From the relay the signal went through an attenuator that was controlled by $\mathrm{E}$, to the Grason-Stadler recording attenuator, and to calibrated earphones, model PDR 10, fitted with MX-41/AR ear cushions. When the waveform generator was turned on, $O$ felt a burst of $60-\mathrm{Hz}$ vibration $200 \mathrm{msec}$. in duration on his left hand, and heard simultaneously a 200-msec. burst of white noise. These signals were presented once per sec. By means of the foot switch, $O$ adjusted the intensity of the white noise to equal the vibratory loudness. The level of white noise in $\mathrm{dB}$ SPL was computed from the recording attenuator and was changed between trials by means of $E^{\prime}$ 's attenuator. The same patterns of vibration as in Experiment I were used.

\section{Results and Discussion}

The mean values of 16 observations per point are shown in Fig. 4. The observed slope of the function relating $\mathrm{AB}$ SPL of white noise to number of vibrators is 15.9, a good approximation to the predicted slope of 15.2. When the number of vibrators was increased from one to five in a pattern, Os increased the level of white noise approximately $10 \mathrm{~dB}$, an increase in the level of

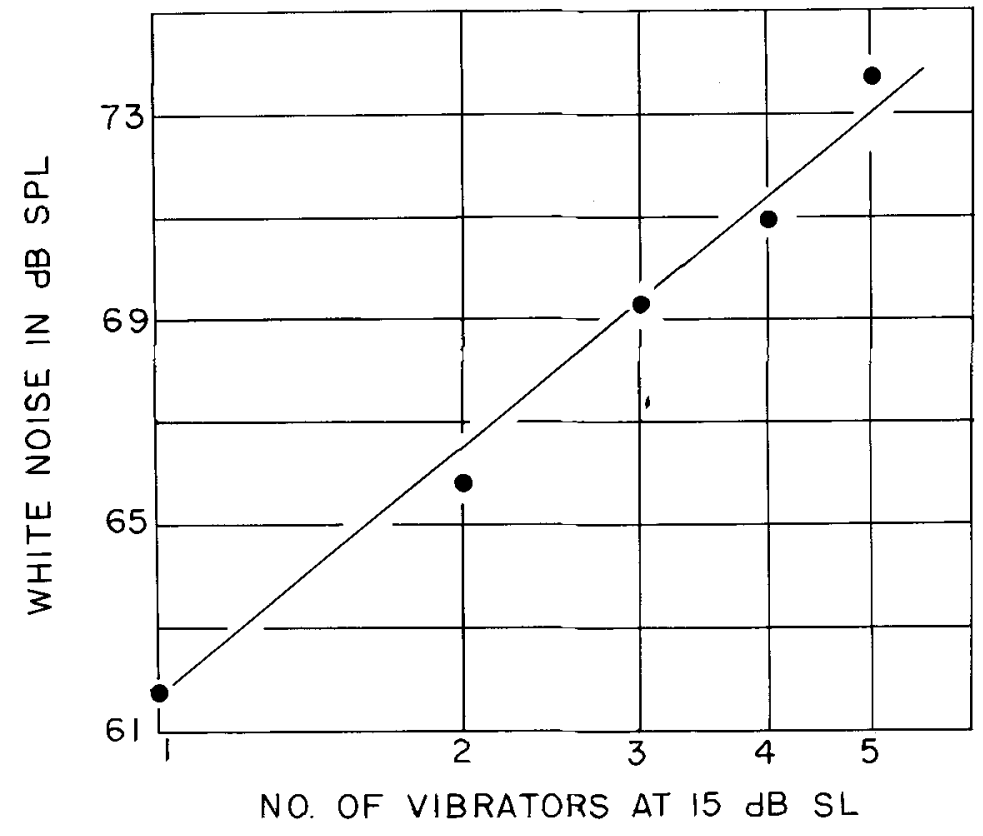

Fig. 4. White noise level needed to match the loudness of different numbers of vibrators on the fingers. 


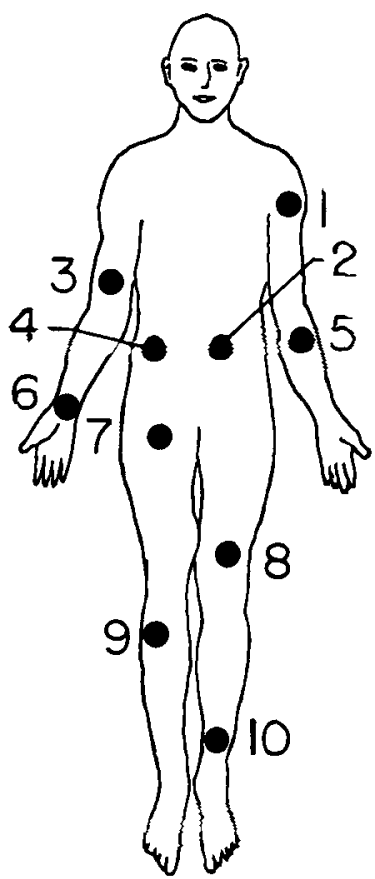

Fig. 5. Positions of the 10 vibrators on the body.

white noise which produced a doubling in the loudness of the white noise (Stevens \& Tulving, 1957). The results of Experiments I and III indicate that it makes little difference whether one uses a match to a single vibrator or to white noise; increasing the number of vibrators in a pattern from one to five results in an approximate doubling of the loudness. This finding lends support to the validity of the matches, as Stevens (1959a) indicated with regard to his cross-modality matches. Moreover, this would help to rule out the possibility that in Experiments I and II the standard vibrator interacted in some subtle way with the test vibrators to influence the matches. Finally, by demonstrating that white noise could be used as a standard, Experiment IV was made possible.

\section{EXPERIMENT IV}

Although the finger tips provide a convenientlocation for cutaneous stimulation, to focus attention exclusively upon them is to neglect possibly more important information. Experiment IV was concerned with the manner in which loudness addition takes, place over the surface of the body and to compare the results with those obtained on the finger tips. Moreover, it was possible to employ a greater number than five vibrators to see what effect this would have.

\section{Apparatus and Procedure}

Five $O s$ were used in this experiment. The apparatus and procedure were the same as in Experiment III with the exceptions as indicated below. In order to fasten them to the body, the test vibrators were mounted on Velcro straps and fitted with contactors $2 \mathrm{~cm}$ in ciameter. The vibrators were placed on the body at the same sites used by Geldard and Sherrick (1965). See Fig. 5.

A Goodmans V -47 vibrator was placed contralaterally to position number eight, and an absolute threshold to a burst of $60-\mathrm{Hz}$ vibration, $200 \mathrm{msec}$. in duration, was obtained by a modified method of limits. The vibrator at position number eight was matched for equal loudness to the $V-47$ vibrator raised to $15 \mathrm{~dB} \mathrm{SL}$. The other vibrators at positions one through seven and nine and 10 were matched one at a time to number eight for equal loudness.

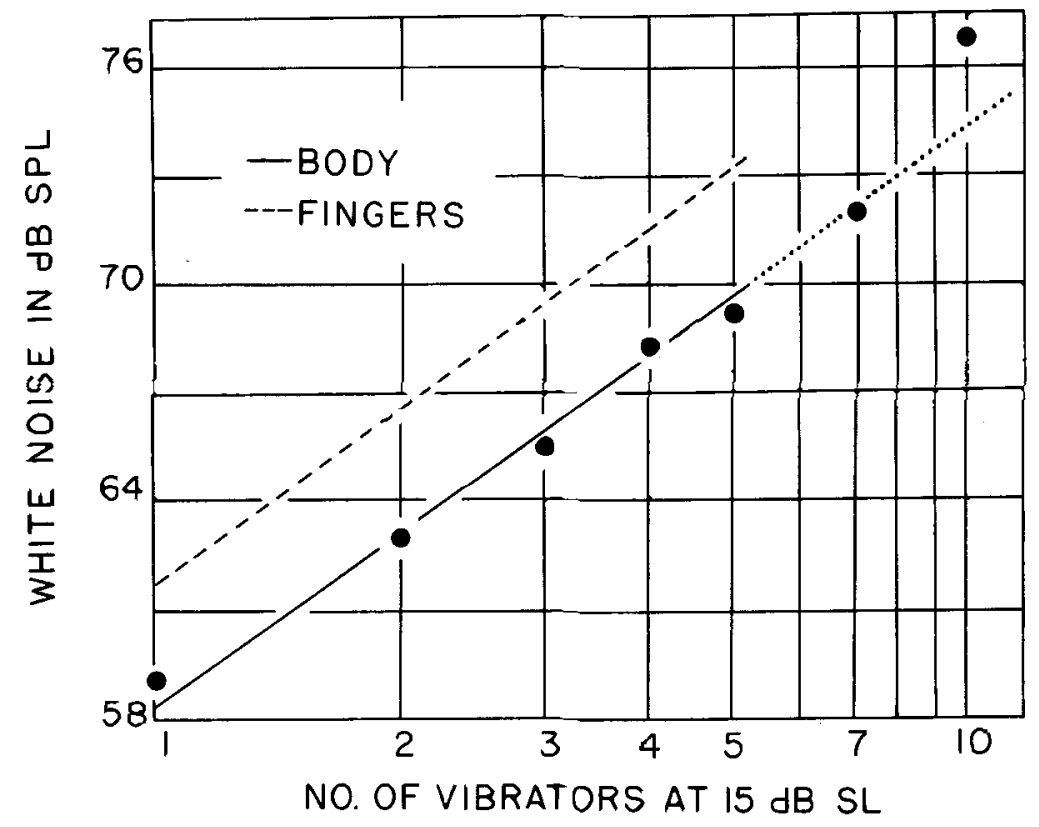

Fig. 6. White noise level needed to match the loudness of different numbers of vibrators on the body. 
When all 10 vibrators were matched, patterns of vibration containing from one to 10 vibrators were presented simultaneously with a burst of white noise. $O$ adjusted the level of white noise until it equalled the pattern of vibration in loudness. The patterns were as follows: vibrator number 5 ; vibrators number 5,8 ; $3,4,7 ; 1,4,7,9 ; 1,2,3,9,10 ; 1,2,3,4,6,8,10 ; 1,2,3$, $4,5,6,7,8,9,10$. The patterns were chosen to maximize the distance among vibrators and to utilize all body loci.

\section{Results and Discussion}

The means of 15 observations per point are presented in a $\log -\log$ plot in Fig. 6. To compare these results with those obtained in Experiment III, a line was fitted to the first five points. The slope of the function relating $\mathrm{dB}$ SPL of white noise to number of vibrators is 16.1 , which does not differ significantly from 15.9, the slope obtained in Experiment III. This slope was extended out to include the values obtained using seven and 10 vibrators. Neither of these mean values differs significantly from the values predicted by the extension of the slope.

Looking at Fig. 6, it is obvious that, despite the many differences between the fing $\epsilon$ rs and the body, such as absolute sensitivity, underlying tissue structure, and density of innervation, vibrotactile loudness addition takes place in nearly the same way on both. The similarity in loudness addition on the body and finger loci agrees with the results obtained using a single vibrator. Békésy $(1960$, p. 566) has shown that within limits a given amplitude of vibration produces nearly the same loudness on the finger tip as it does on the shoulder.

Of the five variables investigated in this study, only one of them, number of vibrators, was a significant factor in loudness addition. None of the four remaining variables (distance between vibrators, loudness levels of components, kind of standard-single vibrator or white noise, or loci on the body) affected loudness addition within the limits studied. It is, of course, possible that, had the distance between stimuli been made small enough or been varied in a systematic manner, some effect on loudness addition might have been evidenced. It is also possible that extremes of intensity might have produced some effect. Thus Békésy (1960, pp. 615-616) found that the growth of vibrotactile loudness was affected only at a critical distance between stimuli, viz., the two point limen, and only at a loudness close to threshold. But the present evidence seems to show that, except for the number of vibratory generating sites, all the factors manipulated in these experiments play an inconsequential role in loudness addition.

\section{References}

Bekésy, G. v. Über die Vibrationsempfindung. Akust. Z, 1939, 4, 315-334.

Bekésy, G. v. Experiments in hearing. New York: McGraw-Hill, 1960.

Fletcher, F., \& Munson, W. A. Loudness, its definition, measurement and calculation. J. Acoust. Soc. Amer., 1933, 5, 82-108.

Geldard, F. A. Pattern perception by the skin. Report of International Symposium on the Skin Senses, Florida State University, Tallahassee, Florida, March, 1966, in press.

Geldard, F. A., \& Sherrick, C. E. Multiple cutaneous stimulation: the discrimination of vibratory patterns. J. Acoust. Soc. Amer., $1965,37,797-801$.

Gibson, R. H. Virginia Cutaneous Project, Progress Report No. 40, 1959 .

Howes, D. H. The loudness of multicomponent tones. Amer. J, Psychol., 1950, 63, 1-30.

Sherrick, C. E. Simple electromechanical vibration transducer. Rev. scient. Instrum., 1965, 36, 1-2.

Spector, P. Virginia Cutaneous Project, Progress Report No. 21, 1953.

Stevens, J. C., \& Tulving, E. Estimations of loudness by a group of untrained observers. Amer. J. Psychol., 1957, 70, 600-605.

Stevens, S. S. Cross-modality validation of subjective scales for loudness, vibration, and electric shock. J. exp. Psychol., 1959a, 57, 201-209.

Stevens, S. S. Tactile vibration: dynamics of sensory intensity. J. exp. Psychol., 1959b, 57, 210-218.

\section{Note}

1. This study was supported, in part, by a grant from the National Institutes of HeaIth, U. S. Department of Health, Education, and Welfare, and in part, by a grant from the National Science Foundation.

(Received in the Editorial Office April 18, 1966.) 